\title{
SÉANCE DU 28 AVRIL 1922
}

PRÉSIDENCE DE M. P. GUÉRIN.

Lecture est donnée du procès-verbal de la précédente séance, dont la rédaction est adoptée.

M. le Président annonce deux nouvelles présentations.

MM. R. Benoist et J. C. Schoute, ayant rempli les conditions prescrites par les statuts, sont nommés membres à vie.

MM. Burollet et Hinglais, récemment admis, ont envoyé une lettre de remerciements à la Société.

L'ordre du jour appelle communication des notes suivantes :

Une Mousse nouvelle pour la France dans la Drôme : Orthotrichum Schawii Wils,, et présence aux environs de Valence des Camptothecium aureum Bry. eur. et Epipterygium Tozeri (Grev.) Lindb.

$$
\text { PAR M. G. DISMIER. }
$$

Orthotrichum Schavii Wils. - Aux environs de Die, à la montagne d'Aucelon, j'ai recueilli, en juillet dernier, dans la forêt du Sapey $(1400 \mathrm{~m}$.) et au pas de la Pousterle (1 $200 \mathrm{~m}$.) sur des trones de Hêtres, quelques touffes de cette rarissime espèce, en bel état de fructification.

Découverte par J. Schaw en juin 1860, sur un Frêne en 
Écosse, cette Mousse fut retrouvée dix ans plus tard par R. Ruthe, le 4 juillet 1870 , sur un Peuplier d'Italie en Allemagne, près de Bärwalde, dans la Marche de Brandebourg. A la suite d'une nouvelle période décennale, Philibert récoltait, en juillet 1881, d'assez nombreux exemplaires d'Orthotrichum Schavii dans les montagnes de la Corse, près de la Foce de Vizzavona, entre 1200 et 15000 mètres, sur des troncs de Hêtres ${ }^{1}$.

Comme aucune localité mondiale n'est venue s'ajouter depuis quarante ans à celle de la Corse, il faut admettre que cette espéce est une haute rareté ou bien qu'elle est méconnue.

J'ai comparé les spécimens que j'ai recueillis à la montagne d'Aucelon avec l'exemplaire (in Herb. Mus. de Paris) de Philibert. La seule différence quej'aie pu constater consiste dans la teinte et l'aspect de la paroi capsulaire : parfois brunâtre et légèrement plissée dans la plante dioise, jaune clair et lisse sur l'échantillon corse.

L'Orthotrichum Schavii n'offre pas beaucoup de difficultés de détermination, je crois cependant devoir rappeler que la plupart des espèces européennes du genre Orthotrichum du groupe à stomates phanéropores ont, après la sporose, les dents du péristome dressées, étalées ou renversées et appliquées étroitement contre la paroi capsulaire. Il n'y a que les quatre espèces suivantes : 0 . leiocarpum, 0 . speciosum, $O$. Schavii, et $O$. Lyelli dont les dents offrent une toute autre disposition à l'état sec : elles sont courbées en are de cercle de manière à ne toucher l'urne que par leur extrème pointe.

L'Orthotrichum Schavii se différencie a priori et tour à tour de chacun de ses trois congénères par les caractères suivants : péristome sans cils, capsules incluses et feuilles dépourvues de propagules. Chez l'Orthotrichum leiocarpum le péristome est double: l'exostome étant accompagné de cils festonnés presque aussi larges que les dents, l' $O$. speciosum a les capsules nettement exsertes et les feuilles de l'O. Lyellii sont abondamment

1. Brotherus (in Engler et Prantl., p. 472,1 ) indique l'Orthotrichum Schavii dans la Haute-Italie : cette indication se rapporte à une erreur de De Notaris qui avait décrit dans son Epilogo l'Orthotrichum Franzonianum sous le nom d'O. Schavii. 
G. Dismier. - UNE MOUSSE NOUVElle POUR LA France.

pourvues de propagules bruns, cylindriques, pluricellulaires et ordinairement visibles à l'œil nu.

Camptothecium aureum Bry. eur. - Cette Mousse, jusqu'à présent, n'était connue que dans les départements côtiers de la Méditerranée. L'été dernier, aux environs de Valence, je l'ai d'abord trouvée près de Montéléger (Drôme) où elle croissait sur un coteau calcaire aride et dénudé en compagnie des Rhacomitrium canescens, Trichostomum crispulum, Pleurochæte squarrosa, Tortula muralis, Ditrichum flexicaule et Cheilothela chloropus; ensuite je l'ai revue à Saint-Péray (Ardèche) sur un talus ensoleillé de la route d'Alboussières.

Le Camptothecium aureum d'après Boulay ${ }^{1}$ est répandu dans les Alpes-Maritimes ${ }^{2}$, le Var, les Bouches-du-Rhône, plus rare dans le Gard, l'Hérault et les Pyrénées-Orientales. Brotherus (l. c., II, p. 1 138) l'indique depuis l'Espagne jusque dans la Syrie et la Palestine et de plus en Algérie. Le Camptothecium aureum est également signalé dans le Portugal, le Maroc et dans plusieurs îles méditerranéennes : Corse, Sardaigne, Sicile, Corfou, etc. En somme, cette espèce est nettement caractéristique du bassin méditerranéen. Comme Montéléger et SaintPéray sont situés presque sous le $44^{\circ} \mathbf{5}^{\prime}$ de latitude Nord, ces deux localités sont les plus septentrionales de l'Europe où cette espèce ait été observée.

\section{Epipterygium Tozeri (Grev.) Lindb. - Cet Epipterygium} Tozeri se rencontre çà et là sur les sables siliceux humides dans les parties inférieures des vallons situés à gauche de la route de Saint-Péray à Cornas (Ardèche).

D'une manière générale, on peut dire que l'Epipterygium Tozeri entoure la France des Alpes-Maritimes à la Manche, sans quitter, à quelques exceptions près, les départements littoraux

1. Boulay (l'abbé), Muscinées de la France, $1^{\text {re }}$ partie, Mousses, p. 138, 1884.

2. Camus (F.), Documents pour la flore bryologique des Alpes-Maritimes (Bull. de la Soc. bot de Fr., 1910, p. CXL). - Dans ce travail M. Camus n'indique le Camptothecium aureum qu'au Golfe-Jouan (Bornet). Dans les recherches que j'ai faites sur le littoral méditerranéen au cours des ànées $1914,1916,1917$ et 1918 je n'ai vu ce, Camptothecium ni à Nice, ni
à Cannes. 
de la Méditerranée et de l'Atlantique et qu'il ne s'écarte guère, entre ces deux mers, du pied des Pyrénées.

L'Epipterygium Tozeri est calcifuge et à mon avis intransigeant. Cette intransigeance donne, tout au moins partiellement, l'explication des interruptions importantes que l'on constate entre les différentes régions où cette Mousse a été observée, principalement sur les côtes méditerranéennes et atlantiques.

Dans les Alpes-Maritimes, où j'ai fait de nombreuses recherches tant à Nice qu'à Cannes, j'ai remarqué que l'on ne commence à trouver l'Epipterygium Tozeri qu'aux environs de Vallauris, c'est-à-dire lorsque apparaissent les gneiss, cette Mousse suit alors le littoral dans la direction de l'Ouest, passe d'abord à Cannes où je l'ai recueillie sur plusieurs points et traverse ensuite les massifs de l'Estérel et du Tanneron : le premier porphyrique, le second gneissique, pour atteindre finalement le Var où MM. Corbière et Jahandiez ${ }^{1}$ l'ont signalée dans plusieurs localités des Maures, massif constitué par des roches cristallophylliennes. Comme le reste de la côte méditerranéenne repose sur des terrains calcarifères, l'Epipterygium Tozeri disparait. En résumé, toute cette première zone correspond, à peu de choses près, à ce que l'on est convenu d'appeler la Cồte d'Azur.

Pour retrouver cette Mousse, il faut aller la chercher dans la Haute-Garonne aux environs de Toulouse d'où elle passe dans les Hautes-Pyrénées, près de Tarbes pour atteindre les BassesPyrénées.

Sur la côte atlantique, Jeanbernat et Renauld ${ }^{1}$ ont indiqué l'Epipterygium Tozeri dans les Landes du Boucau aux dunes du Médoc. Cette espèce a même été trouvée par Motelay ${ }^{2}$ à la pointe de Grave et au Verdon, près de Bordeaux. Au Nord de l'embouchure de la Gironde, toute la Charente-Inférieure est calcique, par suite, nouvelle disparition de l'E. Tozeri. Ce n'est que plus loin, en Vendée, qu'il réapparaît, mais en même temps

1. Corbière (J.) et Jahandiez (E.), Muscinées du département du Var (Ann. de la Soc. d'Hist. nat. de Toulon, 1921, suppl au tome IV, p. 32).

2. Jeanbernat et Renauld (F.j, Guide du bryolog ue dans la chaine des Pyrénées et du Sud-Ouest de la Franse (Rev. de Bot., II, 1883 et 1884, p. $4-7$ et 11). 
que les gneiss, pour se continuer sans interruption sur le sol cristallin de la Bretagne et du Cotentin où il a été observé sur de nombreux points, notamment par MM. Camus et Corbière. Sur tout le reste du littoral de la Manche, qui appartient en majeure partie au système crétacé, l'Epipterygium Tozeri est inconnu.

Exceptionnellement, à l'embouchure de la Loire, il s'avance dans l'intérieur en Maine-et-Loire et même dans la Sarthe, jusqu'aux environs de la Flèche, mais en restant toujours fidèle à l'élément siliceux.

Les mêmes causes produisant des faits analogues, on se trouve amené à envisager la question du substratum comme la raison principale motivant l'absence de l'Epipterygium Tozeri dans la plus grande partie de la vallée du Khône, bien que la région méditerranéenne s'étende presque jusqu'à Valence. Toute cette vallée, depuis la mer jusqu'au-dessus de Tournon (Ardèche), y compris les hauteurs qui l'avoisinent, repose sur le calcaire néocomien, souvent recouvert aux abords du Rhône par des nappes quelquefois très étendues de diluvium alpin, de même \pm calcique. Ce n'est qu'à partir de Beauchastel (Ardèche), en remontant la rive droite, que l'on retrouve en face de Valence et presque en bordure du fleuve les terrains siliceux du Vivarais. La présence de l'Epipterygium Tozeri à Saint-Péray en même temps que l'apparition des granits porphyroïdes dans l'Ardèche vient confirmer l'opinion que je viens d'émettre au sujet de l'influence du substratum. Cette opinion me paraît d'autant plus fondée que Boulay ${ }^{1}$ qui a exploré avec soin, pendant deux années, les environs de Nîmes, fait remarquer que l'Epipterygium Tozeri n'a pas été trouvé sur la rive droite du Rhône. Étant donné que la rive gauche, de Valence à la mer, est de même formation géologique que celle de droite, l' $E$. Tozeri-manque également.

1. Boulay (l'abbé), Études sur la distribution géographique des Mousses en France, au point de vue des principes et des faits, p. 80, 1877. 


\section{$2 \mathrm{BHL}$ Biodiversity Heritage Library}

Dismier, M G . 1922. "Une Mousse nouvelle pour la France dans la Drôme: Orthotrichum Schawii Wils., et présence aux environs de Valence des Camptothecium aureum Bry. eur. et Epipterygium Tozeri (Grev.) Lindb." Bulletin de la Société botanique de France 69, 221-225. https://doi.org/10.1080/00378941.1922.10833429.

View This Item Online: $\underline{\text { https://www.biodiversitylibrary.org/item/93160 }}$ DOI: https://doi.org/10.1080/00378941.1922.10833429

Permalink: https://www.biodiversitylibrary.org/partpdf/161261

\section{Holding Institution}

Missouri Botanical Garden, Peter H. Raven Library

\section{Sponsored by}

Missouri Botanical Garden

\section{Copyright \& Reuse}

Copyright Status: Public domain. The BHL considers that this work is no longer under copyright protection.

This document was created from content at the Biodiversity Heritage Library, the world's largest open access digital library for biodiversity literature and archives. Visit BHL at https://www.biodiversitylibrary.org. 